This item was submitted to Loughborough's Research Repository by the author.

Items in Figshare are protected by copyright, with all rights reserved, unless otherwise indicated.

\title{
Direct deployment of component-based automation systems
}

PLEASE CITE THE PUBLISHED VERSION

http://dx.doi.org/10.1109/ETFA.2012.6489760

PUBLISHER

(c) IEEE

VERSION

AM (Accepted Manuscript)

LICENCE

CC BY-NC-ND 4.0

REPOSITORY RECORD

Kong, Xiangjun, Bilal Ahmad, Robert Harrison, Y. Park, and Leslie J. Lee. 2019. "Direct Deployment of Component-based Automation Systems". figshare. https://hdl.handle.net/2134/12030. 
This item was submitted to Loughborough's Institutional Repository (https://dspace.lboro.ac.uk/) by the author and is made available under the following Creative Commons Licence conditions.

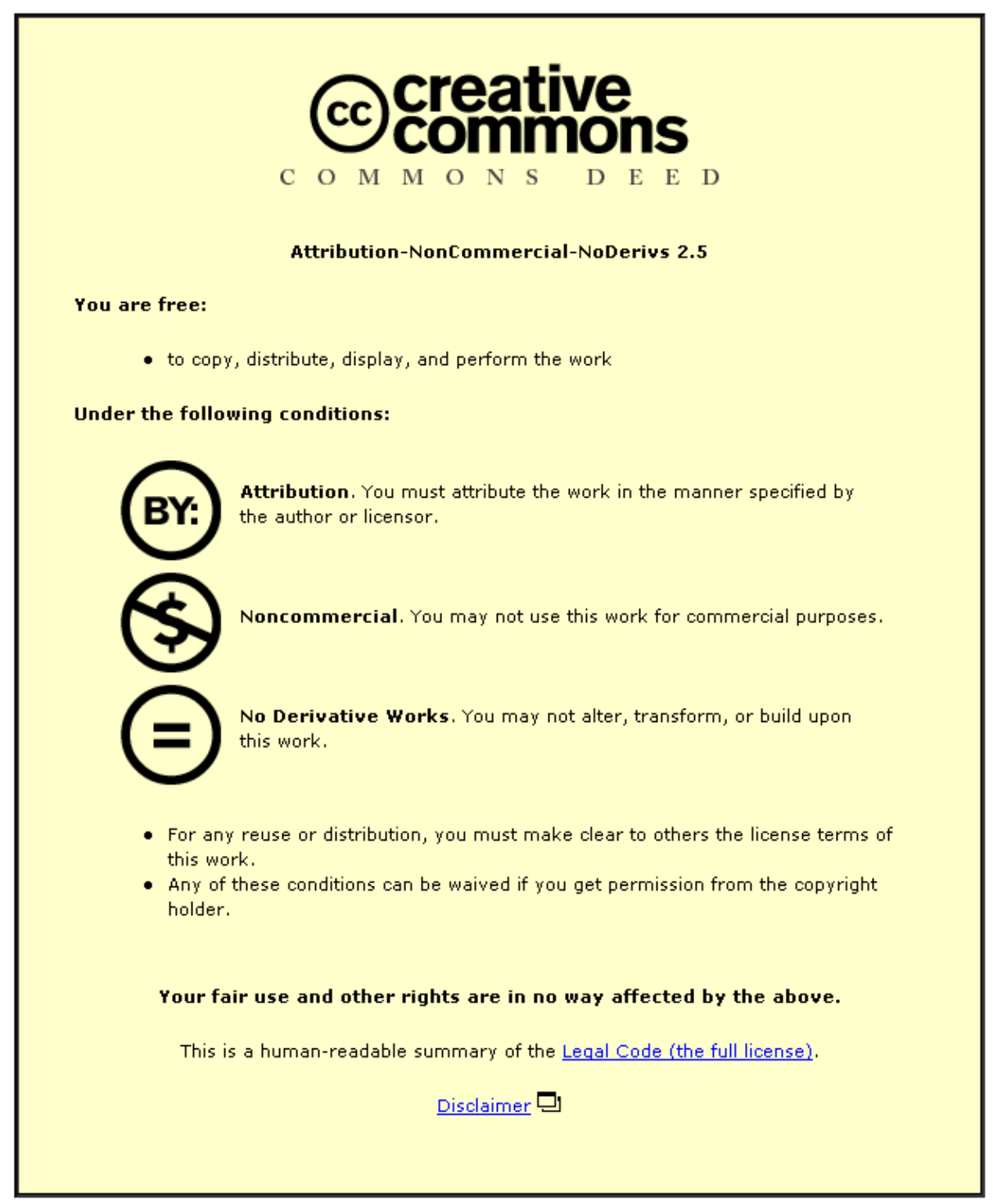

For the full text of this licence, please go to: http://creativecommons.org/licenses/by-nc-nd/2.5/ 


\section{Direct Deployment of Component-Based Automation Systems}

\author{
X.Kong, B.Ahmad, R.Harrison, Y.Park \\ Wolfson School of Mechanical and \\ Manufacturing Engineering \\ Loughborough University \\ Leicestershire, LE11 3TU, UK \\ \{x.kong, b.ahmad, r.harrison, \\ y.park\}@lboro.ac.uk
}

\author{
Leslie.J.Lee \\ Ford Motor Company \\ Powertrain Operations \\ Dunton Engineering Centre \\ Laindon, Essex SS15 6EE, UK \\ 1lee1@ford.com
}

\begin{abstract}
Modular approaches and virtual commissioning are regarded as two key enablers to reduce the effort, cost and time of automation system engineering. This contribution reviews existing researches on the virtual commissioning of modular automation systems. The research work carried out by the authors, which provides a new engineering toolset for the virtual commissioning component-based modular automation system engineering, is reported. Combing the industrial needs related to our research works and the limitations of existing virtual commissioning approaches, an approach to the direct deployment of control systems of component-based automation systems based on virtual commissioning is presented. A use case of the proposed solution using related engineering tools developed by the authors is also provided.
\end{abstract}

\section{Introduction}

Modular approaches and virtual commissioning (VC) are recognised as two key enablers to build reconfigurable automation systems cost effectively and in minimum time. Using a modular approach [1], machine builders are able to build a new system by combining the needed components without the need to understand their complex implementation details [2]. Virtual commissioning enables virtual construction, testing and validation of new production systems prior to their physical build. Through VC, the control logic of automation systems can be verified and improved until it is error free. However, the solutions for directly deploying the validated control logic into real PLCs are still largely missing, which leads to manual PLC programming. Although the programming can be carried out according to the virtually validated control logic, manual programming is still likely to introduce errors.

The research work carried out at Loughborough University, which aims at providing an innovative way of building modular automation systems, is reported in this paper. This research seeks to provide a complete solution for virtual commissioning of modular automation systems and direct deployment of their control systems.

The remainder of this paper is structured as follows: Section 2 summarises related work on VC of modular automation approach. Section 3 reports the research work carried out by researchers from Loughborough University and the needs for direct deployment solutions. Section 4 describes the direct deployment solution proposed by the authors, while section 5 presents a use case.

\section{Related work}

In the last ten years, with the wide utilisation of virtual engineering, many research projects are aiming at virtually building and validating modular automation systems in 3D-based environments to reduce the cost and effort of automation system engineering. These projects commonly break down an automation system into reusable autonomous mechatronic units integrating both mechanical design and control logic. Typical examples are Business Driven Automation (BDA) based on the Component-Based approach proposed [1], Actor-Based Assembly Systems (ABAS) built based on autonomous mechatronic units [3], Modular Machine Design Environment (MMDE) [4] proposed and implemented in VIR-ENG research project and a modular approach for production system engineering by adopting mechatronic objects proposed by researchers from Daimler AG and the University of Magdeburg [5].

Current approaches to build a virtual prototype for virtual commissioning can be classified into Full Simulation of Machinery (FSM) and Hardware-in-theLoop (HIL) simulation [6]. The FSM approach includes a simulation of the production equipment as well as the control hardware itself. This approach can be carried out without any machine hardware. A typical example is the BDA project which develops an engineering toolset for virtual commissioning of component-based automation system. however, the control software can only be tested 
on a pseudo-code basis. In a HIL simulation, on the other hand, the control software can be tested under more realistic conditions by connecting the virtual prototype of a machine to real control hardware, thereby avoiding making changes to the software runtime environment afterwards. However, the PLC codes to be validated mainly need to be programmed manually before performing virtual commissioning. A HIL environment is realised in VIR-ENG project for the design and simulation of component based manufacturing systems.

\section{Research issue}

In the current virtual commissioning approaches, the 3D virtual model building and PLC program development are largely performed separately. By referring to the control logic validated by full simulation, the program can be easier and less error-prone. However, the manual PLC programming is still a repeated work.

The authors are currently working on the research project named Business Driven Automation (BDA) applying the component-based concept in automotive manufacturing sector through collaborative research involving Loughborough University, Ford Motor Company and their machine builders and control vendors. This project aims to enable the realisation of next generation business-driven automation systems which can be readily evolvable under the direct control of the end-user and can be pre-defined in a modular form to enable the majority of process engineering to occur before the beginning of product engineering. Providing a virtual engineering environment for component-based assembly automation systems and a common engineering model that can effectively support the supply chain partners throughout the machine's lifecycle is the main objective of this research. This virtual engineering environment is designed to facilitate the virtual construction, testing and validation of new production facilities prior to their physical build. In this virtual engineering toolset called Core Component Editor (CCE), as shown in Figure 1, a 3D based visualisation environment is provided for prototyping virtual models of components and systems. A virtual logic engine is developed to run the control logic which is described in State Transition Diagram (STD) and validated against the $3 \mathrm{D}$ based virtual models. This engineering toolset is developed based on the component-based approach proposed in [2,7] by Harrison, etc. It provides an innovative way of the FSM of modular automation systems. However, compared to other HIL research projects such as VIR-ENG, the control logic validated in CCE is not real PLC code and manual programming works are still needed.

According to the requirements of the end users and machine builders involved in this research project, the control logic information validated by FSM in CCE should be reused and directly deployed into the real control systems thereby most of the error-prone manual programming works can be avoided. Based on these requirements, the authors propose a direct deployment solution and develop related engineering toolset which will be presented in the following section.

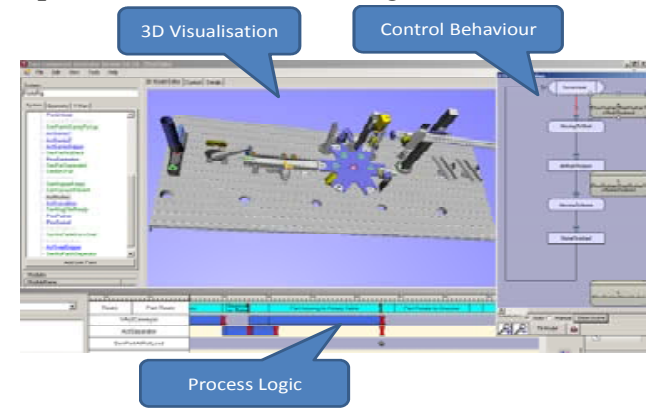

Figure1. 3D based virtual engineering tool-CCE

\section{An approach to direct deployment of PLC control software}

In this section, an innovative approach to the directly deployment of PLC control software for modular automation systems is presented. To enable the direct deployment of PLC control code, a new architecture for the control system of component-based automation system is proposed. An overview of the proposed software architecture and the deployment framework is reported then.

The control software generation method proposed in this paper is essentially based on the use of standard library function blocks and the machine configuration data derived from the virtual simulation tools. The standard library function blocks are a set of generic, prevalidated and reusable PLC code that describes the behaviour of actuators and sensors. While the machine configuration data consist of the hierarchical structure of components within a manufacturing cell and the embedded control behaviour of each component.

To realise the direct deployment of control software from the machine configuration data and standard function blocks, a new PLC control software architecture is proposed by the authors. As illustrated in Figure 2, this control software is composed of the following parts:

- Data blocks for control logic information: the states, transitions and conditions of each component are described as user defined data type and finally saved as arrays of corresponding derived data types.

- Function block: The functionalities of a function block include 1) changing state according to the command from logic engine and translating the command into output $\mathrm{I} / \mathrm{O}$ variables to drive the real machine component, and 2) reporting the current state to the logic engine according the input I/O variables. 
- Logic Engine: is a function which monitors all the array of components, states, transitions and conditions. It keeps scanning all those arrays and changes the values of corresponding items when their related values change at runtime.

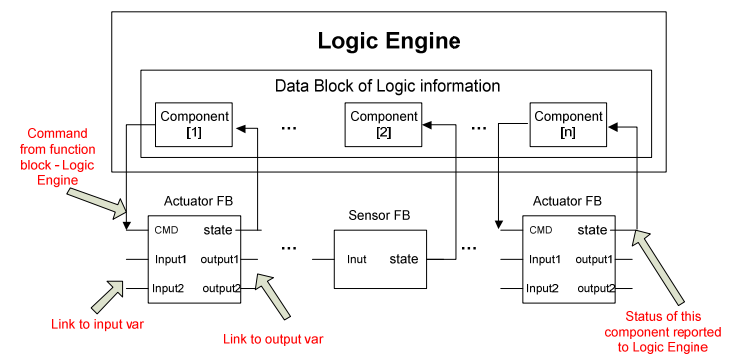

Figure 2. Control system of a componentbased automation system

All the control logic information is monitored by the logic engine while the function blocks are only used for translating I/O information and communicating it with the Logic Engine. The Logic Engine, as well as all the function blocks, is reusable and the control logic information is directly derived from $\mathrm{VC}$ tools.

A engineering tool has been developed by the authors to implement the proposed direct deployment solution. As shown in Figure 3, the system architecture of the engineering tools is as following:

Data management layer: for manipulating the input and output data. The input data includes reusable function blocks, I/O variables and control logic information exported from virtual commissioning tools. The output data is the executable PLC code which can be downloaded into real PLCs directly without any manual modifications.

User interface layer: is used to do the I/O mapping and allocate related function blocks to components.

Deployment function layer: is used to generate different PLC codes for different type of PLCs by combining the reusable data and I/O mapping information from the user interface layer.

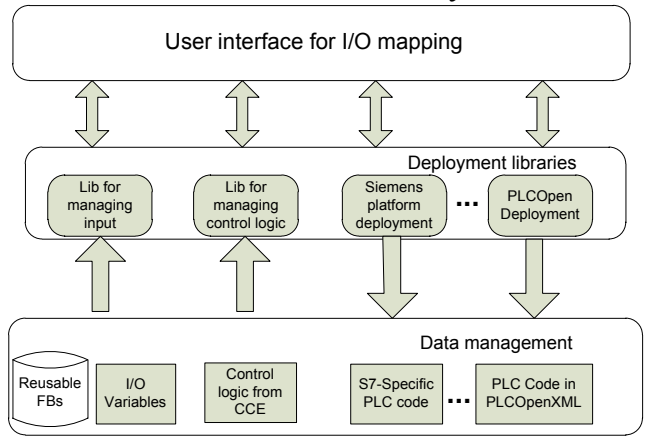

Figure 3. System architecture of the
software for direct deployment

In the proposed direct deployment approach, all the function blocks are developed and tested during the component building phase. The control logic information consisting of control behaviour of components and process sequences are built and validated in 3D-based virtual commissioning tools. To generate the complete control software, the only manual work need to be done is the $\mathrm{I} / \mathrm{O}$ mapping.

\section{Use case}

This section presents the evaluation of the approach to modular automation system engineering described in this paper. First, a test rig was virtually modelled and validated. Then, the PLC code for this rig was developed using both the conventional approach and the direct deployment approach, or a Siemens S7 target controller.

\subsection{Virtual system development and validation}

To perform the full virtual commissioning, the test rig, as shown in Figure 4, was virtually built in two steps, namely component development and system development.
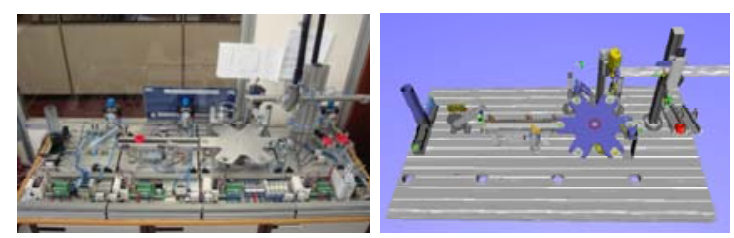

Figure 4. Real test rig and virtual test rig

The test rig is composed of 14 actuator components and 13 sensor components. Firstly, all the needed components and their corresponding function blocks need to be built. Different components might use the same function block if they have the have similar state behaviour. For example, all the sensor components correspond to the same function block and the component pusher, the component swivel arm and other 4 actuators actually correspond to the same function blocks. An example of the pusher component is shown in Figure 5.

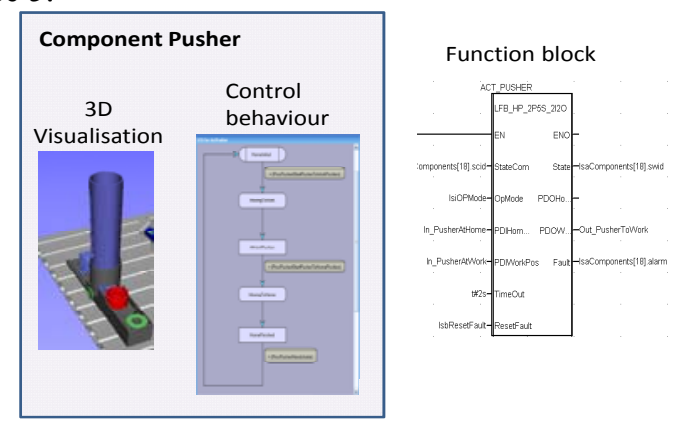

Figure 5. A component and the related FB

The virtual system of the test rig can be built by assembling related components and adding process logic. The process logic is essentially a state-transition diagram defining the required sequence of operations. 
The control logic interlocking of involved components when building a system can be referred to [7].

\subsection{Direct deployment}

In direct deployment, the control behaviours of all the components and process are described as arrays of user defined data type (UDT) in S7. The logic engine is described in S7-SCL programming language to monitor all the arrays of logic information. In this approach, the functionality of a function block is to translate the commands from the Logic Engine into output variables, and to translate the input variables and report these to the Logic Engine. The control system is thus composed of the Logic Engine function block plus the function blocks for the actuators or sensors which need to communicate with the physical I/O of the PLC, as shown in Figure 6.

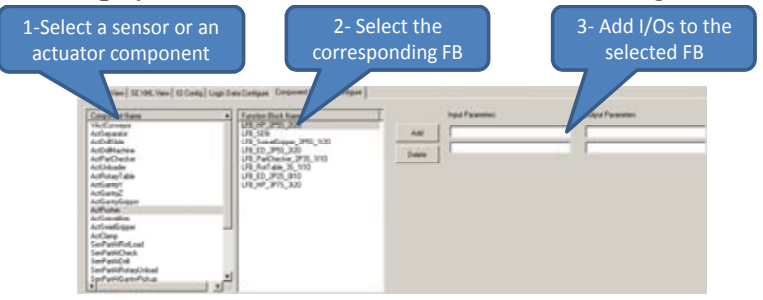

Figure 6. I/O mapping with user interface

Since all these types of function block can be predefined, no manual programming is required. Obviously where new functionality is needed additional function blocked can be added to the reuse library. After the I/O mapping for related function blocks in the user interface of CCE toolset, the whole source code for S7 platform can be generated automatically. The simplified structure of the PLC program generated is shown in Figure 7.

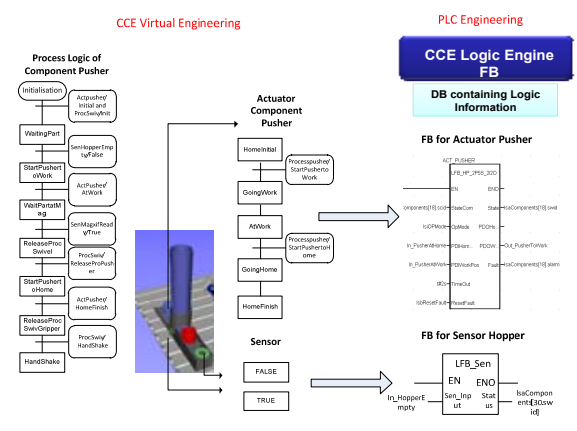

Figure7. Component-based control programs

\section{Conclusions}

The research work carried out at Loughborough University provides an innovative virtual engineering approach and a corresponding engineering tool to the full simulation and direct deployment of modular automation systems. The most significant advantage of the solution presented in this paper is that it provides an approach to automatically generate well-structured PLC control software for different PLCs. The other advantages include: 1) the modelled components can be reused and reconfigured to achieve various machine configurations, and 2) virtual machine prototypes will be highly portable as the data has been saved in an open data format. The $3 \mathrm{D}$ based virtual engineering toolset has been used for virtual modelling and virtual commissioning of powertrain assembly systems for the end user Ford. The authors now are working on development of the engineering tool for control logic deployment. A prototype version has been completed and used to perform tests against a test rig and is now being used for the direct deployment of real machines for the end user.

Future research work will be realising the direct deployment of control logic for other more platforms such as open standard PLCOpenXML.

\section{Acknowledgements}

The authors gratefully acknowledge the support of the Engineering and Physical Sciences Research Council (EPSRC) and our industrial collaborators through the Innovative Manufacturing and Construction Research Centre (IMCRC) and BDA project in carrying out this research.

\section{References}

[1] Harrison, R., et al., "Reconfigurable modular automation systems for automotive power-train manufacture", International Journal of Flexible Manufacturing Systems, 2006. 18(3): p. 175-190.

[2] Lee, S., et al., "A component-based approach to the design and implementation of assembly automation system", Proceedings of the Institution of Mechanical Engineers, Part B: Journal of Engineering Manufacture, 2007. 221(5): p. 763-773.

[3] Martinez Lastra, J.L., "Reference mechatronic architecture for Actor-based Assembly Systems", 2004, Tampere : Tampere University of Technology.

[4] Moore, P.R., et al., "Virtual engineering: an integrated approach to agile manufacturing machinery design and control", Mechatronics, 2003. 13(10): p. 1105-1121.

[5] M. Bergert, J.K., "Mechatronic Data Models in Production Engineering", 10th IFAC Workshop on Intelligent Manufacturing Systems. 2010: Lisbon, Portugal.

[6] Reinhart, G. and G. Wünsch, "Economic application of virtual commissioning to mechatronic production systems", Production Engineering, 2007. 1(4): p. 371379.

[7] Lee, S., et al., "A component-based control system for agile manufacturing", Proceedings of the Institution of Mechanical Engineers, Part B: Journal of Engineering Manufacture, 2005. 219(5): p. 123-135. 